\title{
Optimal Piezoelectric Actuators and Sensors Configuration for Vibration Suppression of Aircraft Framework Using Particle Swarm Algorithm
}

\author{
Quanzhen Huang, ${ }^{1,2}$ Suxia Chen, ${ }^{3}$ Huayan Pu, ${ }^{2}$ and Ning Zhang ${ }^{1}$ \\ ${ }^{1}$ School of Electrical Information Engineering, Henan Institute of Engineering, Henan 451191, China \\ ${ }^{2}$ School of Mechatronics Engineering and Automation, Shanghai University, Shanghai 200072, China \\ ${ }^{3}$ School of Computer, Henan Institute of Engineering, Henan 451191, China \\ Correspondence should be addressed to Huayan Pu; phygood_2001@shu.edu.cn
}

Received 6 February 2017; Revised 16 April 2017; Accepted 27 April 2017; Published 15 June 2017

Academic Editor: Xiao-Qiao He

Copyright (C) 2017 Quanzhen Huang et al. This is an open access article distributed under the Creative Commons Attribution License, which permits unrestricted use, distribution, and reproduction in any medium, provided the original work is properly cited.

\begin{abstract}
Numbers and locations of sensors and actuators play an important role in cost and control performance for active vibration control system of piezoelectric smart structure. This may lead to a diverse control system if sensors and actuators were not configured properly. An optimal location method of piezoelectric actuators and sensors is proposed in this paper based on particle swarm algorithm (PSA). Due to the complexity of the frame structure, it can be taken as a combination of many piezoelectric intelligent beams and L-type structures. Firstly, an optimal criterion of sensors and actuators is proposed with an optimal objective function. Secondly, each order natural frequency and modal strain are calculated and substituted into the optimal objective function. Preliminary optimal allocation is done using the particle swarm algorithm, based on the similar optimization method and the combination of the vibration stress and strain distribution at the lower modal frequency. Finally, the optimal location is given. An experimental platform was established and the experimental results indirectly verified the feasibility and effectiveness of the proposed method.
\end{abstract}

\section{Introduction}

Numbers and locations of piezoelectric sensors and actuators are of great significance for piezoelectric smart structure, vibration control performance, system implementation costs, and so on $[1,2]$.

There are a few problems for configurations of sensors or actuators in the progress of promoting the engineering application. When they are placed at improper locations, this will lead to much more uncontrolled modal response messages in sensor signals and actuators may inspire uncontrolled modal responses, which will make observation or control spillover or even make the control system unstable. In fact, it is an optimization problem to determine the best locations of sensors and actuators. And many scholars have made some profound studies on this issue. These studies can be split into two: on the one hand, those that determine the optimal allocation criterion, namely, optimizing objective function. On the other hand, those that choose the appropriate optimization methods. For example, Caruso et al. [3] aimed at maximizing the modal controllability and observability of the structure and the locations of piezoelectric patches with a fixed size were optimized. Kumar and Narayanan $[4,5]$ worked out the optimal location of sensor-actuator pairs which were placed in the piezoelectric cantilever plate on the basis of a linear quadratic regulator (LQR). The LQR performance index was used as the objective function of the optimization progress, which was solved by genetic algorithm (GA). Rao et al. [6] proposed particle swarm based evolutionary optimization technique for optimal placement of piezoelectric patch actuators and accelerometer sensors to suppress vibration. Viswamurthy and Ganguli $[7,8]$ proposed a response surface based optimization method for actuator and sensor placement. Manning [9] proposed a two-stage 
optimization strategy for active member placement and strut cross section and compensator parameter optimization for intelligent trusses. T.-W. Kim and J.-H. Kim [10] optimized the placement of the piezoelectric path of the flexible plates by applying the sequential quadratic programming method. Gao [11] investigated integrated optimization of the actuator location and feedback gain in PZT smart trusses with stochastic structural parameters using a two-step optimization strategy. Xian [12] combined a layered strategy and an approximation concept and formed a two-level, branched and multipoint approximation strategy for adaptive truss actuator placement optimization. Liu and Lin [13] took a twolevel optimization method based on a simulated annealing algorithm to determine the optimal channel distribution and the optimal channel voltage for dynamic shape control of structures using piezoelectric materials. Honda et al. [14] employed the placement of piezoelectric actuators, the layup configurations of laminated composite plates, and the $\mathrm{H} 2$ vibration control system as design variables and optimized them simultaneously by GA. Dutta et al. [15] considered artificial bee colony and glowworm swarm optimization algorithms, to find the optimal locations of actuators/sensors and feedback gains of a cantilevered beam.

A piezoelectric aluminum alloy smart frame structure is taken as an experimental model in this paper. Since the structural shape and constraints are complex, the entire framework is taken as a combination of a plurality of piezoelectric intelligent beams and L-shaped structures. Firstly, piezoelectric cantilever is taken as a structural vibration control object. Optimization objective function is established according to controllability and observability criteria of system. Configuration of sensors and actuators is optimized by particle swarm algorithm. Secondly, according to the above optimization process and the analysis results by ANSYS, the final optimal configuration is obtained. An experimental platform was established and the experimental results indirectly verified the feasibility and effectiveness of the proposed method.

\section{Optimization Principle of the Piezoelectric Sensors and Actuators}

According to Ahari [16], the Partial Differential Equation (PDE) of the cantilever beam which is made of aluminum alloy can be described as follows:

$$
\begin{aligned}
F(p, t)= & M(p) \frac{\partial^{2} w(p, t)}{\partial t^{2}} \\
& +2 \zeta[M(p) L]^{1 / 2}\left[\frac{\partial w(p, t)}{\partial t}\right] \\
& +L[w(p, t)] .
\end{aligned}
$$

Here $F(p, t)$ is the distribution of external force; $w(p, t)$ is deflection of beam, which is a function of a space variable $p \in D$ and time $t ; L$ is the hardness distribution of the system, which is a partial differential function about a relative space coordinate $p$ and $M(p)$ is a mass density function, which is a positive definite function about the location of $p$. Here, $M(p)=1, L[w(p, t)]=(E I / \rho A)\left(\partial^{4} / \partial x^{4}\right)$.
The principle for the distribution of the actuators should ensure its influence on the structural perturbation to the greatest degree. In other words, the actuator should transport the energy as large as possible to the structural mode. According to this principle, the criteria for the optimization of the actuators are [17]

$$
o p z^{\prime}=2\left(\sum_{i=1}^{n} E_{i}\right) \sqrt[n]{\prod_{i=1}^{n} E_{i}}
$$

$E_{i}$ is mathematical expectation of the $i$ th modal total energy. It can be considered as two parts: the first part is about the total energy of the system. Generally speaking, some low modal energy is chosen on the basis that modal energy reduces sharply along with the increasing of modal numbers; the other part is considered as the volume of an ellipsoid. The ellipsoid is $n$-dimensional, and its radius is directly proportional to each modal energy.

As the size of the diagonal elements of observability Gramian is directly proportional to the stability of the system, the criteria of the optimal sensor placement are

$$
o p z^{\prime}=\left(\sum_{J=1}^{2 n} \lambda_{J}\right) \sqrt[2 n]{\prod_{J=1}^{2 n}\left(\lambda_{J}\right) .}
$$

$\lambda_{J}$ is the eigenvalue of observability Gramian. The eigenvalue is fundamentally the same as that of controllability Gramian for some small damping structural system, so it is the best situation for the same numbers and positions of sensor and actuator.

\section{Sensor/Actuator Optimization Algorithm Based on Particle Swarm}

3.1. Establishment of Optimization Objective Function. Based on the position optimization guidelines in Section 2, it can be seen that the controllability Gramian gives the relevance of system state and the output and the observability Gramian gives the relevance of the system state and input. But these matrices and eigenvalues are taken depending on the state vector; if the state vector changes, the above will not be established. The above guidelines also show that the vectors of the controllability and observability Gramian are related to optimization configuration criteria; and the controllability and observability Gramian are, respectively, related to matrices $B$ and $C$, so matrices $B$ and $C$ are, respectively, related to the positions of the actuator and sensor.

Therefore, the optimization criterion is to seek the best position to make features of the controllability and observability Gramian optimal. To this end, establish the following best guidelines: when its mathematical value is small, the system is not controllable or not considerable; when the mathematical value is large, the system is controlled or considerable. The value is maximum, and it is the best location.

Before setting the optimal objective function, the observability matrix $W_{\text {obs }}$ and the controllability matrix $W_{\text {con }}$ are, 
TABLE 1: The relevant parameters of sensors/actuators.

\begin{tabular}{lcccccc}
\hline Type & $\begin{array}{c}\text { Length } \\
(\mathrm{mm})\end{array}$ & $\begin{array}{c}\text { Width } \\
(\mathrm{mm})\end{array}$ & $\begin{array}{c}\text { Thickness } \\
(\mathrm{mm})\end{array}$ & $\begin{array}{c}\text { Coupling } \\
\text { coefficient } \\
K_{p}\end{array}$ & $\begin{array}{c}\text { Piezoelectric } \\
\text { constant } \\
d_{31}\end{array}$ & $\begin{array}{c}\text { Bulk density } \\
P\left(10^{3} \mathrm{~kg} / \mathrm{m}^{3}\right)\end{array}$ \\
\hline P-51 & 20 & 6 & 0.4 & 0.62 & 186 \\
PZT-5H & 40 & 8 & 1 & 0.68 & 275 \\
\hline
\end{tabular}

respectively, obtained by the expression between Gramian matrix and the system modal energy [18].

$$
\begin{aligned}
& W_{\mathrm{obs}}=\operatorname{diag}\left(\frac{c_{n}}{4 \xi_{i} w_{i}}, \frac{c_{n}}{4 \xi_{i} w_{i}}\right) \quad i=1,2, \ldots, n \\
& W_{\text {con }}=\operatorname{diag}\left(\frac{\beta_{n}}{4 \zeta_{i} w_{i}}, \frac{\beta_{n}}{4 \zeta_{i} w_{i}}\right) \quad i=1,2, \ldots, n .
\end{aligned}
$$

Here, $\xi$ is the extraneous signal, $\zeta$ is the control signal, and $w$ is the deflection of beam.

If the natural frequency distribution of structural vibration system is well and the damping coefficient is small, the optimal objective function is given as follows:

$$
\operatorname{Obj}=\frac{\operatorname{trace}(W) \sqrt[2 n]{\operatorname{det}(W)}}{\sigma\left(\lambda_{i}\right)}
$$

where $W$ is Gramian matrix, its value is $W_{\text {obs }}$ or $W_{\text {con }}, \sigma\left(\lambda_{i}\right)$ is standard deviation for the eigenvalues $\lambda_{i}$ of Gramian matrix $W, \sqrt[2 n]{\operatorname{det}(W)}$ is the geometric mean of the eigenvalues, its physical significance is the volume of the ellipse, $n$ is the coefficient of freedom degree, $\sigma\left(\lambda_{i}\right)$ is the location mainly avoiding both great and small eigenvalues, and $\operatorname{trace}(W)$ is the output energy of the actuator.

$W$ can be expressed as each order modal energy or modal value; maximum and minimum values of trace $(W)$, $\sqrt[2 n]{\operatorname{det}(W)}$, and $1 / \sigma\left(\lambda_{i}\right)$ have synchronization. And trace $(W)$ may represent sum of each modal energy; a small value can be ignored during the total sum, so that it can be expressed as sum of lower modal energies. $\sqrt[2 n]{\operatorname{det}(W)}$ can be expressed as product of the modal energies, as we all know that each plays a role in the product operation; it means that all modals are functioning, also taking into account higher-order modals. Therefore, this criterion well considers each order modal; it will be very effective to optimize the configuration for the sensor/actuator.

3.2. Process and Analysis of PSO. According to the characteristics analysis of piezoelectric aluminum alloy beam structure, optimization objective function is obtained for the piezoelectric sensor and actuator. As in literature [2], on the basis of finite element modeling, it is studied on the actuator position optimization of dimensional flexible plate using controlled Gramian matrix. In the process of position optimization using controllability and observability Gramian, each iteration's calculations need to solve Lyapunov equations; its computation will see a sharp rise when the system has a large degree of freedom or many actuators need to be position-optimized, resulting in a very long computing time. Thus, in order to avoid complex calculations, particle swarm algorithm was proposed in this paper to optimize the objective function, to achieve advantages of high computational efficiency, fast convergence, and being simple and versatile.

Optimization process of piezoelectric sensor/actuator by particle swarm algorithm broadly is divided into several steps:

(1) First, establish kinetic equations according to optimization object; expressions of each order's natural frequencies and modal strain are obtained.

(2) Carry out modal strain analysis, and get each order's natural frequencies, modal shapes, and modal stress distribution.

(3) The given modal values and the natural frequency are converted to the needed form of the objective function optimization and then are substituted into the objective function, the position based on particle swarm algorithm is optimized, and ultimately an optimal layout plan of piezoelectric sensor/actuator is given.

3.2.1. Modal Analysis of Smart Piezoelectric Beams. Since it is more complex to analyze directly piezoelectric frame structure modal, in order to more clearly describe the optimization methods in the paper, the constituent units of the piezoelectric frame structure (i.e., piezoelectric beam) are directly analyzed. The material of the framework is aluminum alloy, and its related parameters are as follows: length $l=$ $1500 \mathrm{~mm}$, width $b=20 \mathrm{~mm}$, height $h=5 \mathrm{~mm}$, density $\rho=2700 \mathrm{~kg} / \mathrm{m}^{3}$, Young's modulus $E_{p}=7.0 \times 10^{10} \mathrm{~Pa}$, and Poisson's ratio $v=0.33$. Material type of piezoelectric sensors is P-51, material type of piezoelectric actuator is $\mathrm{PZT}-5 \mathrm{H}$, and its parameters are shown in Table 1 [19].

Suppose piezoelectric beam elements are the ideal state; according to (1), combining with the actual situation described above, the dynamic equations and boundary conditions of aluminum beam are obtained as follows:

$$
\begin{aligned}
\frac{E I}{\rho A} & \frac{\partial^{4} y(x, t)}{\partial x^{4}}+2 \zeta \sqrt{\frac{E I}{\rho A}}\left[\frac{\partial^{3} y(x, t)}{\partial x^{2} \partial t}\right]+\frac{\partial^{2} y(x, t)}{\partial t^{2}} \\
& =\frac{1}{\rho A} \sum_{l=1}^{P} \delta\left(x-x_{l}\right) f_{l}(t),
\end{aligned}
$$




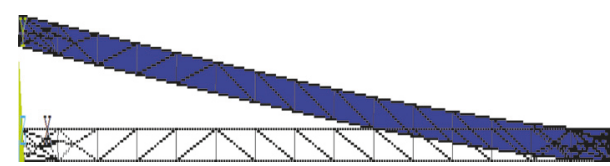

(a) The first order

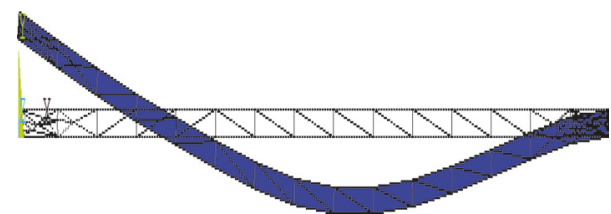

(b) The second order

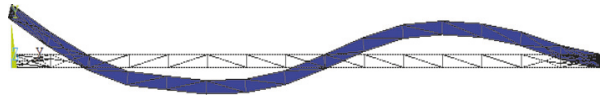

(c) The third order

FIGURE 1: The first 3 vibration modal diagrams.

where $E$ is a modal amount and $I$ is a cross-section secondary distance; it can be converted to the form of the general solution:

$$
\begin{gathered}
y(x)=C_{1} \sin (\beta x)+C_{2} \cos (\beta x) \\
+C_{3} \sinh (\beta x)+C_{4} \cosh (\beta x) \\
\left.y(x, t)\right|_{x=0}=0 \\
\left.\frac{\partial y(x, t)}{\partial x}\right|_{x=0}=0 \\
\left.E I \frac{\partial^{2} y(x, t)}{\partial x^{2}}\right|_{x=l}=0, \\
\left.E I \frac{\partial^{3} y(x, t)}{\partial x^{3}}\right|_{x=l}=0 .
\end{gathered}
$$

Equations (8) and (9) are, respectively, geometric boundary conditions of fixed end and the force boundary conditions of the free end; it can be obtained by the two equations:

$$
\begin{aligned}
& C_{1}=-C_{3}, \\
& C_{2}=-C_{4} \\
& C_{1}[\cos (\beta l)+\operatorname{ch}(\beta l)]+C_{2}[\sin (\beta l)+\operatorname{sh}(\beta l)]=0 \\
& -C_{1}[\sin (\beta l)-\operatorname{sh}(\beta l)]+C_{2}[\cos (\beta l)+\operatorname{ch}(\beta l)]=0 .
\end{aligned}
$$

Conditions of the nontrivial solution for $C_{1}$ and $C_{2}$ in (10) are as follows:

$$
\left|\begin{array}{cc}
\cos (\beta l)+\operatorname{ch}(\beta l) & \sin (\beta l)+\operatorname{sh}(\beta l) \\
-\sin (\beta l)+\operatorname{sh}(\beta l) & \cos (\beta l)+\operatorname{ch}(\beta l)
\end{array}\right|=0 .
$$

Expanding from (11), we can obtain

$$
\cos (\beta l) \operatorname{ch}(\beta l)+1=0 .
$$

The solution for (12) is

$$
\begin{aligned}
& \beta_{1} l=1.875, \\
& \beta_{2} l=4.694, \\
& \beta_{3} l=7.855,
\end{aligned}
$$

TABLE 2: The first 6 natural frequencies of piezoelectric aluminum beams.

\begin{tabular}{lcccccc}
\hline Mode & 1 & 2 & 3 & 4 & 5 & 6 \\
\hline $\begin{array}{l}\text { Natural } \\
\text { frequency (Hz) }\end{array}$ & 29.518 & 34.851 & 95.873 & 178.932 & 200.689 & 253.306 \\
\hline
\end{tabular}

$$
\begin{gathered}
\beta_{4} l=10.996, \\
\beta_{5} l=14.137, \\
\vdots \\
\beta_{n} l \approx\left(\frac{(2 n-1)}{2}\right) \pi .
\end{gathered}
$$

Its natural frequency of each order is

$$
\omega_{n}=\left(\beta_{n} l\right)^{2} \sqrt{\frac{E I}{\rho l^{4}}} \quad n=1,2,3, \ldots, n .
$$

From the above equation, the modal functions of each order are as follows:

$$
\begin{aligned}
\phi_{n}(x)= & \cos \left(\beta_{n} x\right)-\operatorname{ch}\left(\beta_{n} x\right) \\
& +\xi_{n}\left[\sin \left(\beta_{n} x\right)-\operatorname{sh}\left(\beta_{n} x\right)\right] .
\end{aligned}
$$

According to the material parameters of aluminum alloy, using ANSYS software for modal analysis, the first 3 vibration modal diagrams are shown in Figure 1 and the first 6 natural frequencies' table is shown in Table 2.

3.2.2. Position Optimization Analysis of Piezoelectric Sensor/Actuator. To clearly describe the optimization methods, establish the coordinate position schematic diagram of the piezoelectric aluminum beams, as shown in Figure 2, where $X$ coordinate system represents the length of the beam (the actual length of the beam is $l=1500 \mathrm{~mm}$; a small cell represents $50 \mathrm{~mm}$ ) and the $Y$ coordinate system represents the width of the beam (since the actual width $b=20 \mathrm{~mm}$, a small cell represents $4 \mathrm{~mm}$ ); for example, the coordinates of 
TABLE 3: The numbers and position optimization table of sensors/actuators.

\begin{tabular}{|c|c|c|c|c|c|c|c|c|}
\hline Number & & & & ition coo & & & & \\
\hline 1 & $(1,3)$ & & & & & & & \\
\hline 2 & $(1,3)$ & $(27,3)$ & & & & & & \\
\hline 3 & $(1,3)$ & $(5,4)$ & $(27,2)$ & & & & & \\
\hline 4 & $(1,3)$ & $(5,3)$ & $(20,3)$ & $(27,3)$ & & & & \\
\hline 5 & $(1,3)$ & $(8,3)$ & $(16,2)$ & $(22,3)$ & $(28,3)$ & & & \\
\hline 6 & $(1,2)$ & $(4,3)$ & $(10,3)$ & $(16,4)$ & $(24,2)$ & $(27,3)$ & & \\
\hline 7 & $(1,3)$ & $(3,3)$ & $(8,2)$ & $(15,4)$ & $(19,4)$ & $(23,2)$ & $(28,3)$ & \\
\hline 8 & $(1,4)$ & $(3,2)$ & $(7,2)$ & $(13,4)$ & $(17,2)$ & $(21,4)$ & $(24,3)$ & $(28,3)$ \\
\hline
\end{tabular}

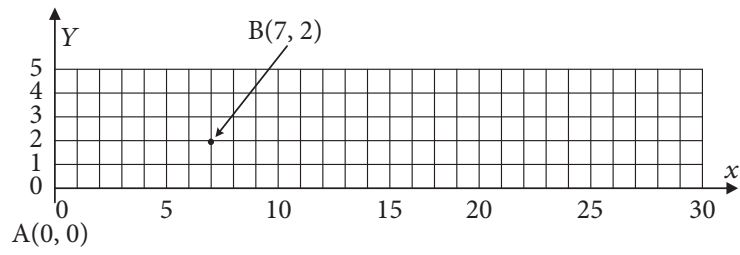

FIGURE 2: Coordinate establishment schematic of piezoelectric aluminum beams.

point $B$ are $(7,2)$ in Figure 3 ; the actual relative position from point $A$ is $(350 \mathrm{~mm}, 8 \mathrm{~mm})$.

Aluminum beam positions of 1-8 pairs of sensor/actuator are optimized, respectively, by particle swarm algorithm, the coordinate position is shown in Figure 2, the whole PSO process is shown in Figure 3, the evolution curve of the PSO fitness is shown in Figure 4, and, finally, optimum position table of the sensor/actuator is shown in Table 3.

\section{Modal Analysis of Aircraft Frame Structure}

In order to verify the proposed method, the model structure is simplified to be an aluminum frame structure and the spring constraint is applied to the four corners as shown in Figure 5.

The frame structure is mainly composed of aluminum alloy sheet and the spring for connecting. The main performance parameters are the elastic modulus of $7 \times 10^{10} \mathrm{~Pa}$, density of $2700 \mathrm{~kg} / \mathrm{m}^{3}$, Poisson's ratio of 0.3 , and elastic coefficient of the spring of $140 \mathrm{n} / \mathrm{m}$. A high-end 3D 10-node solid structure unit is chosen using finite element emulation software ANSYS and the spring uses COMBIN14 unit with axial or torsional performance. The first 10 order modal frequencies and mode shapes are got by modal analysis on the simulated spacecraft frame only with the spring constraints, of which the first 6 order mode shapes are rigid displacements and the 7th to 10th order are vibration modals as shown in Figure 6. Their corresponding modal frequencies are $12.25 \mathrm{~Hz}, 24.35 \mathrm{~Hz}, 33.60 \mathrm{~Hz}$, and $59.80 \mathrm{~Hz}$.

ANSYS analysis is only for the main frame model. Model wing is composed on the main frame structure, in order to achieve the purpose of the whole piezoelectric intelligent framework. The density is $1730 \mathrm{~kg} / \mathrm{m}^{3}$, Young's modulus is $2.0 \times 10^{10} \mathrm{~Pa}$, and Poisson's ratio is 0.16 . The first 10 modal analyses are carried out for experimental model structure;
TABLE 4: The first 4 order natural modal frequencies.

\begin{tabular}{lcccc}
\hline $\begin{array}{l}\text { The order of vibration } \\
\text { modal }\end{array}$ & 1 & 2 & 3 & 4 \\
\hline $\begin{array}{l}\text { Frequency (not attaching } \\
\text { piezoelectric sheets)/Hz }\end{array}$ & 19.58 & 33.20 & 48.87 & 61.75 \\
$\begin{array}{l}\text { Frequency (attaching } \\
\text { piezoelectric sheets)/Hz }\end{array}$ & 20.70 & 34.35 & 49.70 & 62.50 \\
\hline
\end{tabular}

due to limited space, we now give the first 2 modal strain distributions shown in Figures 7 and 8.

The first 4 order natural modal frequencies are shown in Table 4.

Based on optimization method in Section 3.2, taking into account the stress distribution of each vibration modal and other regions with the relatively concentrated distribution, the whole consideration to carry out piezoelectric sensor/actuator arrangement is shown in Figure 9 with $10 \times$ 10 channel.

\section{FXLMS Algorithm [20]}

The FXLMS control algorithm structure is shown in Figure 10. $P(z)$ is the primary path from exciter to error sensor. $H(z)$ is the second path from the secondary source to the error sensor.

The control system has $M$ controllers and $N$ sensors. $X(k)$ is input vector, which is the reference signal. $Y(k)$ is the output vector of the $M$ th controller in time $k . \widehat{Y}(k)$ is $L$ th control response vector, and $B(k)$ is $L$ th external response vector. $E(k)$ is $L$ th cancellation residual vector. $\widehat{X}(k)$ is $X(k)$ filtered by $\widehat{H}(z) . \widehat{H}(z)$ is the identified model of $H(z)$. If every element in $\widehat{H}(z)$ is described using a $P$ th order filter, and each controller is an $N$ th order filter, $W$ is the $(M \times N)$ th controller weights matrix. The order of the reference signal is $N$.

The adaptive filtered feedforward FXLMS algorithm is shown as follows:

$$
\begin{aligned}
Y(k) & =W(k) X(k) \\
E(k) & =B(k)+\widehat{X}(k) W(k) \\
W(k+1) & =W(k)-\mu \widehat{X}^{T}(k) E(k) .
\end{aligned}
$$




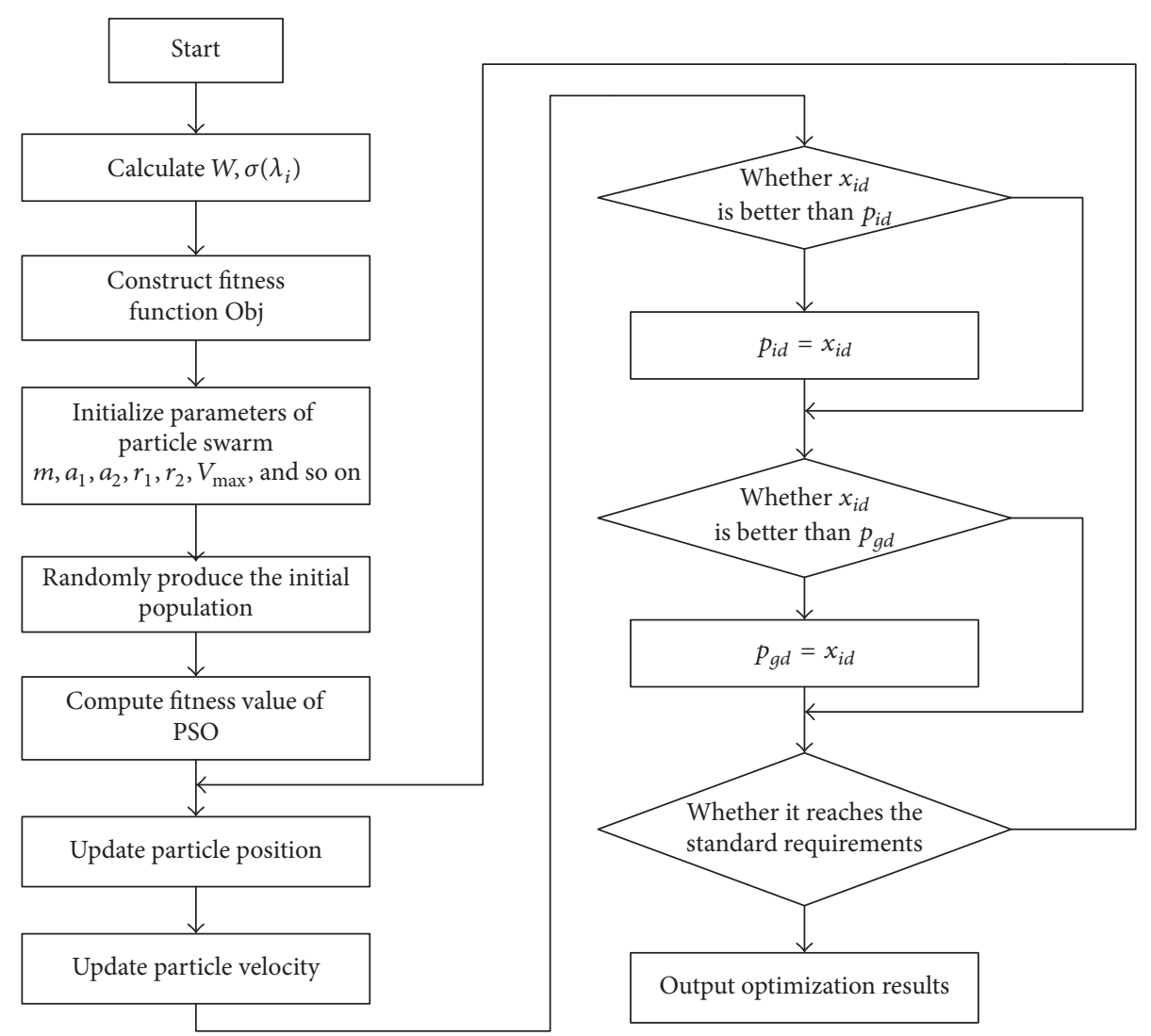

FIGURE 3: Position optimization algorithm flowchart of PSO piezoelectric element.

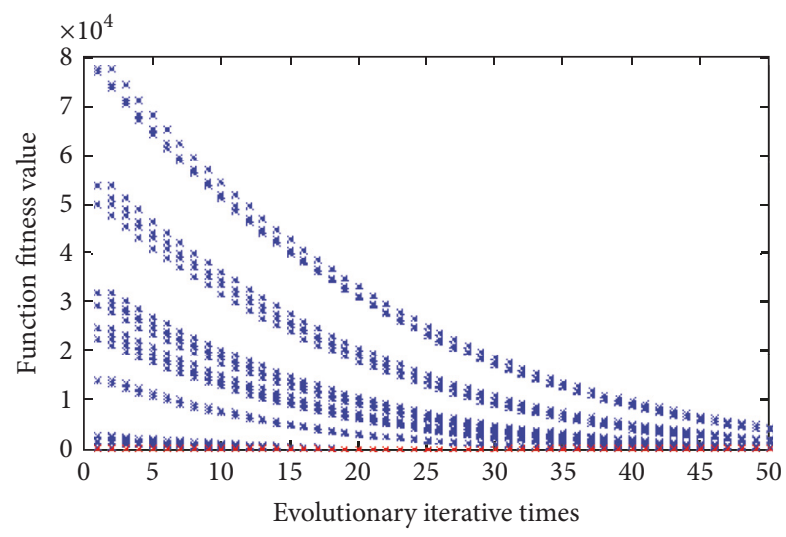

Figure 4: The evolution curve of PSO fitness.

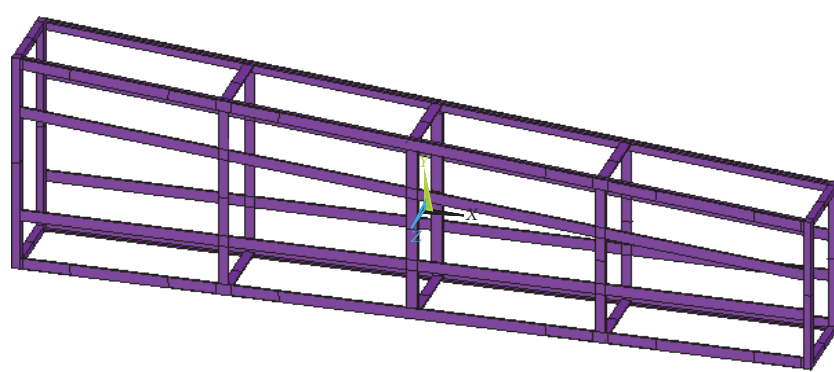

FIGURE 5: Simplified model of aircraft framework.
Here, $\mu$ is the step factor. $0<\mu<1 . W(k)$ is the $(M \times N)$ th controller weights matrix. $\widehat{X}(k)$ is $X(k)$ filtered by $\widehat{H}_{2}$.

$$
\begin{gathered}
W(k)=\left[\begin{array}{cccc}
w_{10}(k) & w_{11}(k) & \cdots & w_{1 N-1}(k) \\
w_{20}(k) & w_{21}(k) & \cdots & w_{2 N-1}(k) \\
\cdots & \cdots & \ddots & \cdots \\
w_{M 0}(k) & w_{M 1}(k) & \cdots & w_{M N-1}(k)
\end{array}\right] \\
\widehat{X}(k)=\left[\begin{array}{cccc}
\widehat{X}_{11}(k) & \widehat{X}_{12}(k) & \cdots & \widehat{X}_{1 L}(k) \\
\widehat{X}_{21}(k) & \widehat{X}_{22}(k) & \cdots & \widehat{X}_{2 L}(k) \\
\cdots & \ldots & \widehat{X}_{m l}(k) & \cdots \\
\widehat{X}_{M 1}(k) & \widehat{X}_{M 2}(k) & \cdots & \widehat{X}_{M L}(k)
\end{array}\right],
\end{gathered}
$$

where $\widehat{X}_{m l}(k)=\left[\widehat{x}_{m l}(k), \widehat{x}_{m l}(k-1), \ldots, \widehat{x}_{m l}(k-N+1)\right], \widehat{x}_{m l}(k-$ $n+1)=\sum_{i=0}^{P-1} h_{m l i} x(k-i-n+2)$.

\section{The Vibration Control Experiment and Verification}

Experimental platform mainly consists of the aluminum outer frame for the stationary support, signal generator 


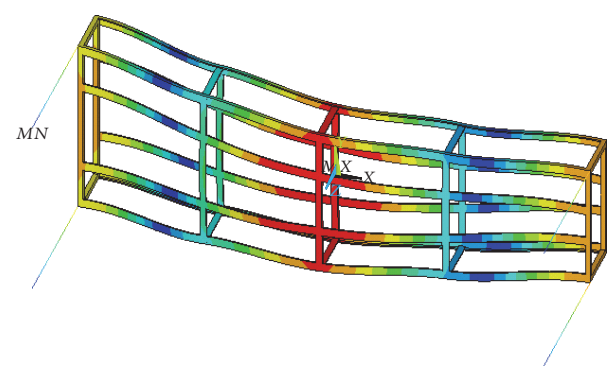

(a) The 7th-order modal strain plot

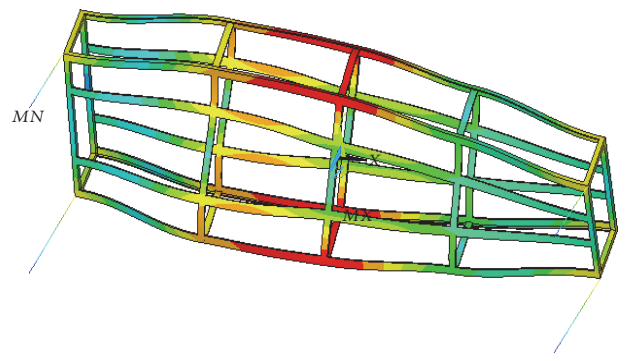

(c) The 9th-order modal strain plot

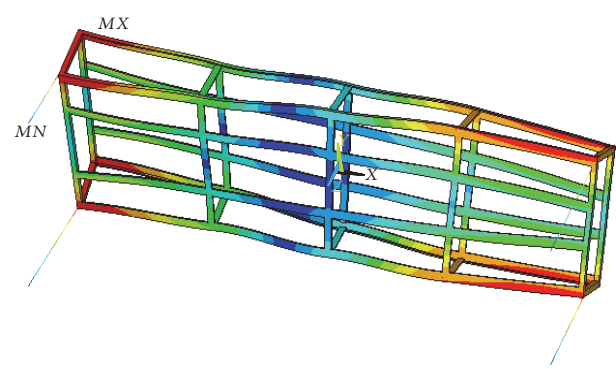

(b) The 8th-order modal strain plot

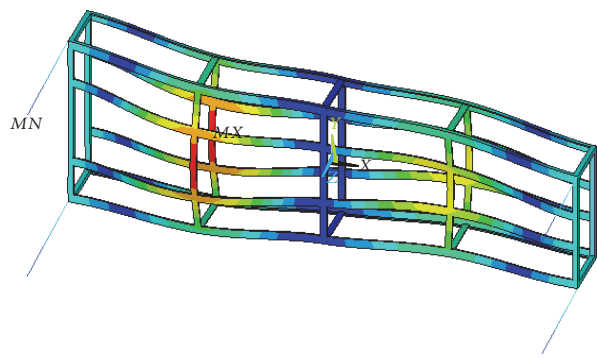

(d) The 10th-order modal strain plot

FIgURE 6: Modal strain plot of the 7th to 10th order.

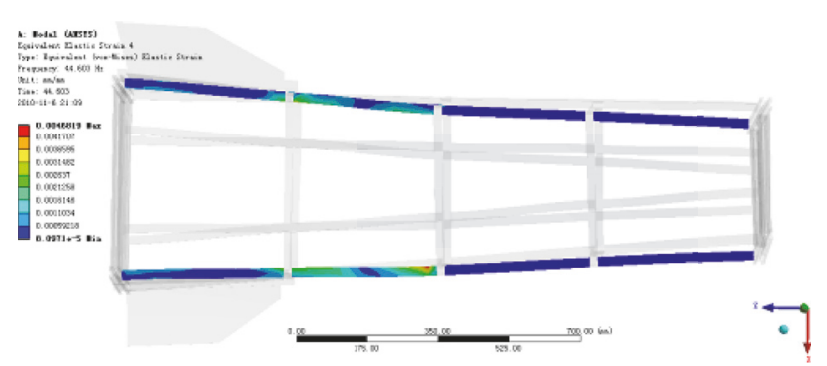

(a) The main support frame

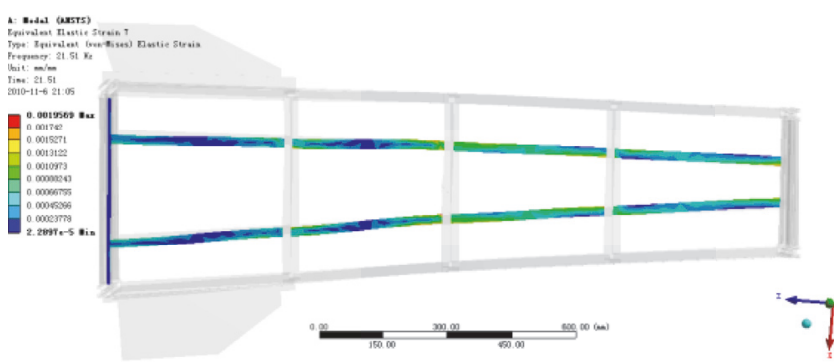

(b) The auxiliary support frame

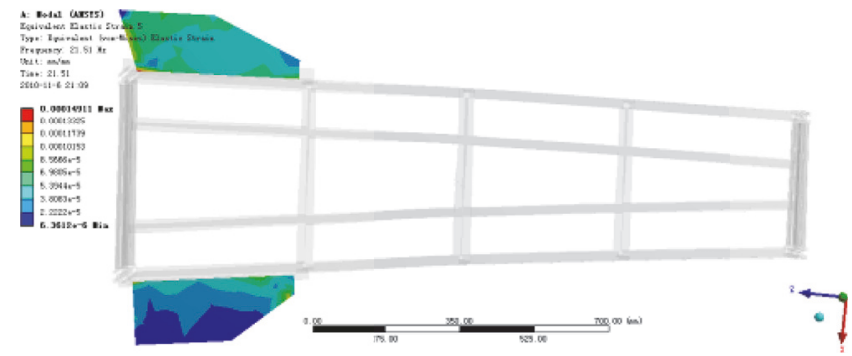

(c) The level wing

FIGURE 7: The first-order vibration modal strain distribution.

(Agilent 33521A, a $30 \mathrm{MHz}$ function/arbitrary waveform generator), a signal amplifier (model 2210), a power amplifier (model 7602M wideband amplifier), a charge amplifier (YE5852A), a low pass filter (YE3760), high-speed data AD acquisition card (PIC 1712) and DA output card (PIC 1721), a high performance computer, an oscilloscope, and related measurements and control units. The measurement and control software is developed on VC 6.0 to guarantee the realization of the system function and the control process. The vibration control experimental platform is shown in Figure 11.

Multichannel vibration control experiment of 10 inputs and 10 outputs is carried out. The experimental process roughly includes the following steps: 


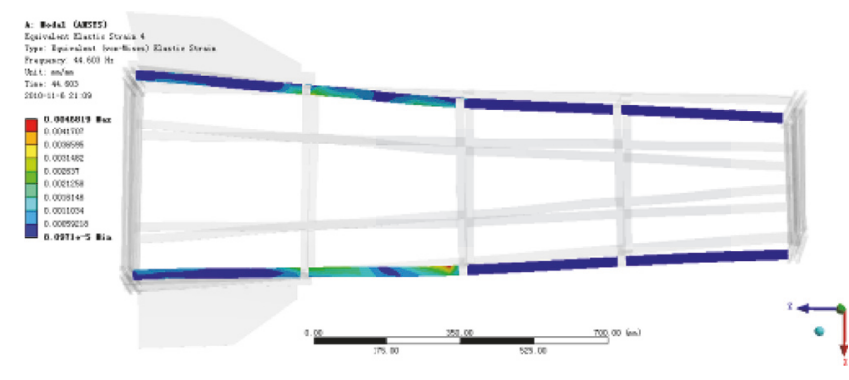

(a) The main support frame

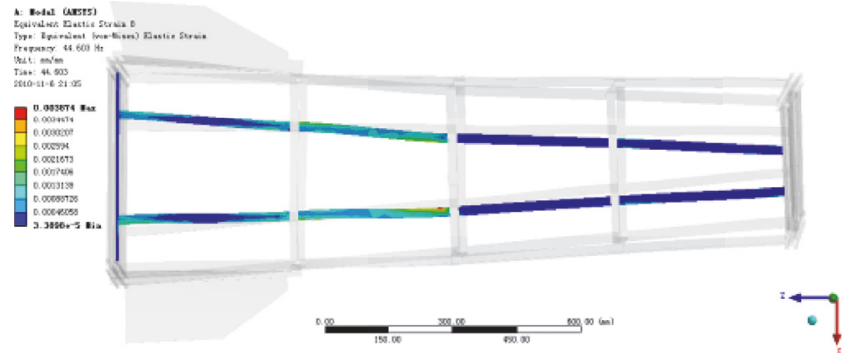

(b) The auxiliary support frame

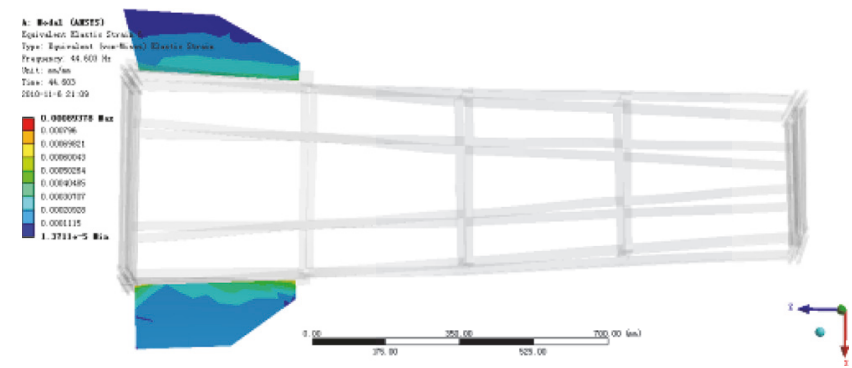

(c) The level wing

FIGURE 8: The second-order vibration modal strain distribution.

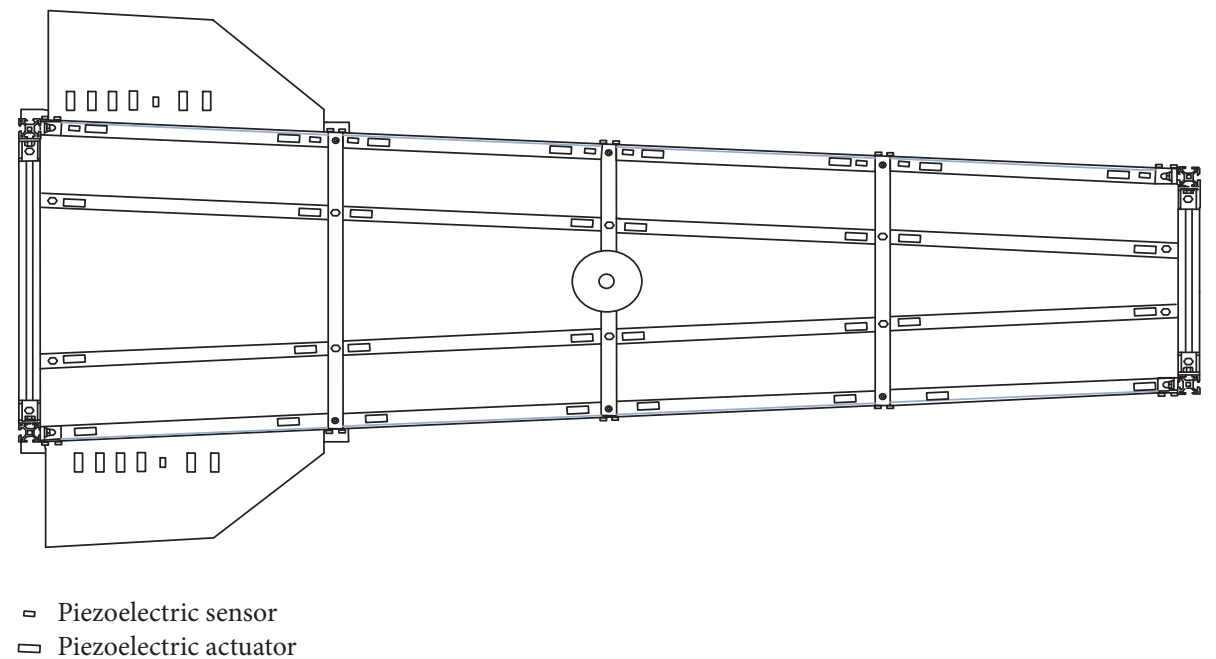

FIGURE 9: Planform of the sensor/actuator location arrangement.

(1) Output a sinusoidal stimulating signal whose frequency is $f=20.70 \mathrm{~Hz}$ using the signal generator. The signal is amplified by the power amplifier and passed the vibration exciter, which generates the continual vibration to the frame.

(2) Vibration control experiment is carried out by FXLMS. Based on the measure and control procedure, set the control parameters, such as sample frequency of $f_{c}=300 \mathrm{~Hz}$, the step convergence of $\mu=0.01$, and the length of the adaptive filter of $N=24$ factors.
(3) Run control algorithm and store the timed data, which is needed to prepare for the experimental analysis.

Figures 12(a) and 13(a), respectively, show vibration response time histories for channels 2 and 8. Figures 12(b) and 13(b), respectively, show control voltage outputs for channels 2 and 8. It can effectively suppress the vibration response of the model structure at about $3.5 \mathrm{~S}$. Figure 14 is structural vibration response overall suppression effect diagram (i.e., the sum of each sensor signal minimum mean square error (MSE)). Structural overall response is significantly decreased 


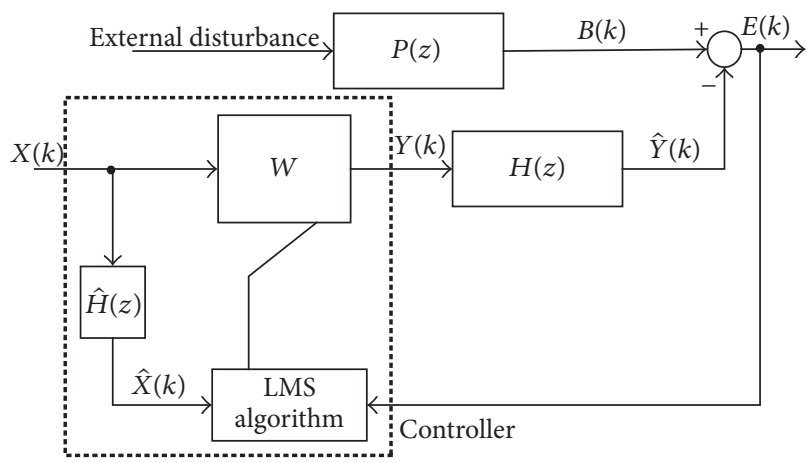

FIGURE 10: Structural diagram for classical FXLMS algorithm.

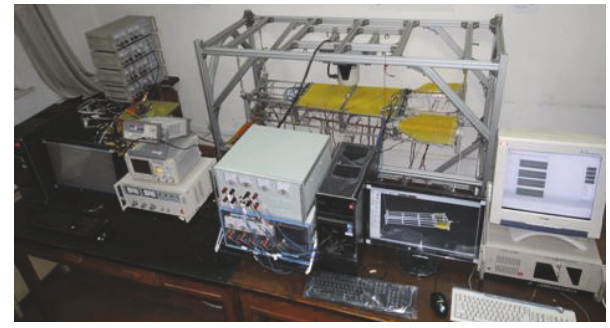

FIGURE 11: The vibration control experimental platform.

after running the control, indicating that the overall vibration is good.

Although the experiment uses the external disturbance signal simulated by the single-frequency signal on controlled structure, the actual vibration response of controlled structure not only has the frequency of the excitation signal but also contains a large number of harmonic components. There are two reasons for the resulting phenomenon: First, it is caused by the coupled response of the vibration modal structure; second, it is caused by the way of bonding of piezoelectric patches and the impure excitation signal. Figure 15 shows power comparison for the overall structure vibration before and after control. It can be seen from the figure that the amplitude of the excitation frequency has been significantly reduced. Additionally, from the standpoint of noise control, while suppressing the structure vibration, the noise caused by structure vibration radiation also reduces. Individual component response of harmonic frequency has been enhanced after control, which is caused by the digital controller quantized noise and the piezoelectric drive itself.

In order to verify the superiority of the algorithm proposed in this paper, the genetic algorithm (GA) and particle swarm optimization algorithm are used to optimize the objective function. And the sensor and actuator configuration scheme is established. The vibration control experimental platform is established. Using the same vibration
TABLE 5: Comparison of vibration suppression rate with that of different optimization algorithm.

\begin{tabular}{lcccc}
\hline & Channel 1 & Channel 5 & Channel 10 & $\begin{array}{c}\text { Total } \\
\text { channels }\end{array}$ \\
\hline$\eta V$ (GA) & $80.3 \%$ & $78.6 \%$ & $84.2 \%$ & $80.5 \%$ \\
$\eta V$ (PSA) & $56.4 \%$ & $54.9 \%$ & $60.8 \%$ & $58.2 \%$ \\
\hline
\end{tabular}

control algorithm, the vibration control experiment data are analyzed. And the vibration suppression effect is shown in Table 5, in which $\eta V=\left(Z V_{1}-Z V_{2}\right) / Z V_{1} \times 100 \%$, where $\eta V$ is for suppressing the vibration rate, $Z V_{1}$ is the vibration amplitude before control, and $Z V_{2}$ is the vibration amplitude after control; according to Table 5, it can be concluded that the proposed algorithm has better vibration control effect.

\section{Conclusion}

The locations of sensors and actuators have large impact on the control system performance. Therefore, an optimal allocation method of piezoelectric sensor and actuator is proposed based on particle swarm algorithm in the paper. An experimental platform was established and the experimental results indirectly verified the feasibility and effectiveness of the proposed method. Comparison of vibration suppression rate with that of different optimization algorithm shows that genetic algorithm is better than particle swarm algorithm for optimal configuration problem of piezoelectric actuators and sensors. The method proposed in this paper is limited to configuration of distributed sensors and actuators.

\section{Conflicts of Interest}

All authors declare that there are no conflicts of interest regarding the publication of this paper. 


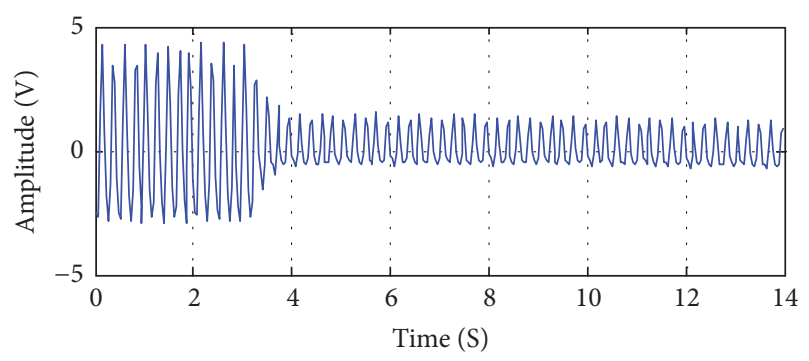

(a)

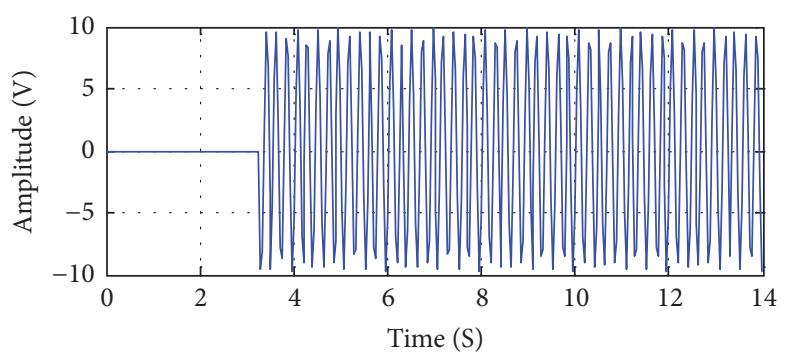

(b)

FIGURE 12: (a) Time history of the vibration response of channel 2. (b) Time history of the control voltage output of channel 2.

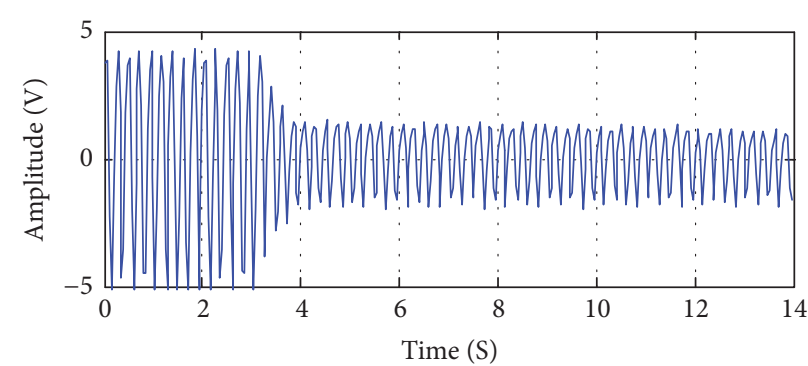

(a)

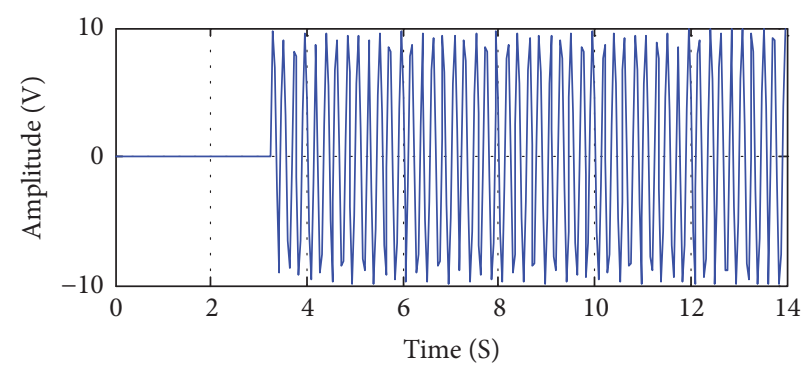

(b)

Figure 13: (a) Time history of the vibration response of channel 8. (b) Time history of the control voltage output of channel 8.

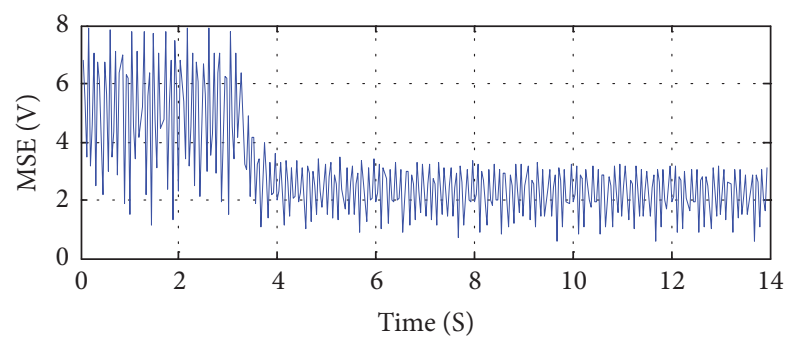

FIGURE 14: Effect diagram of the total structure vibration response.

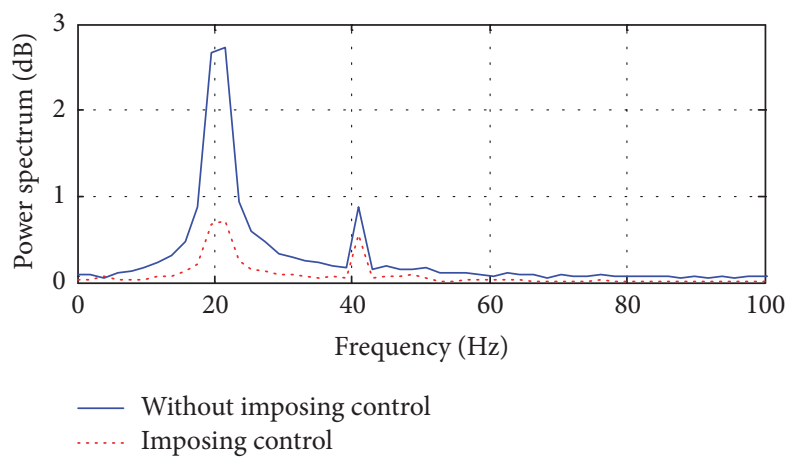

FIGURE 15: Comparison diagram of structural vibration power spectrum without control and imposing control.

\section{Acknowledgments}

This research is supported by the National Nature Science Foundation of China (no. 61403123, no. 51575329, and no. 61525305), Technological Innovation Talents Projects of Henan Universities (no. 17HASTIT020), The Science and Technology Project, Henan Province (no. 162102210099, no. 162102310081, and no. 172102210205), Youth Backbone Teacher Project, Henan Province (no. 2016GGJS153), and Shanghai Rising-Star Program (no. 17QA1401500).

\section{References}

[1] M. I. Frecker, "Recent advances in optimization of smart structures and actuators," Journal of Intelligent Material Systems and Structures, vol. 14, no. 4-5, pp. 207-216, 2003.

[2] I. Bruant, L. Gallimard, and S. Nikoukar, "Optimal piezoelectric actuator and sensor location for active vibration control, using genetic algorithm," Journal of Sound and Vibration, vol. 329, no. 10, pp. 1615-1635, 2010.

[3] G. Caruso, S. Galeani, and L. Menini, "On actuators/sensors placement for collocated flexible plates," in Proceedings of the 11th Mediterranean Conference on Control and Automation, p. 6, Rhodes, Greece, 2003.

[4] K. R. Kumar and S. Narayanan, "The optimal location of piezoelectric actuators and sensors for vibration control of plates," Smart Materials and Structures, vol. 16, no. 6, pp. 26802691, 2007.

[5] K. R. Kumar and S. Narayanan, "Active vibration control of beams with optimal placement of piezoelectric sensor/actuator pairs," Smart Materials \& Structures, vol. 17, no. 5, pp. 1726-1731, 2008.

[6] A. K. Rao, K. Natesan, M. S. Bhat, and R. Ganguli, "Experimental demonstration of $H_{\infty}$ control based active vibration suppression in composite fin-tip of aircraft using optimally placed 
piezoelectric patch actuators," Journal of Intelligent Material Systems and Structures, vol. 19, no. 6, pp. 651-669, 2008.

[7] S. R. Viswamurthy and R. Ganguli, "Optimal placement of trailing-edge flaps for helicopter vibration reduction using response surface methods," Engineering Optimization, vol. 39, no. 2, pp. 185-202, 2007.

[8] S. R. Viswamurthy and R. Ganguli, "Optimal placement of piezoelectric actuated trailing-edge flaps for helicopter vibration control," Journal of Aircraft, vol. 46, no. 1, pp. 244-253, 2009.

[9] R. A. Manning, "Optimum design of intelligent truss structures," in Proceedings of the 32nd Structures, Structural Dynamics, and Materials Conference, pp. 528-533, Baltimore,MD,U.S.A., April 1991.

[10] T.-W. Kim and J.-H. Kim, "Optimal distribution of an active layer for transient vibration control of a flexible plate," Smart Materials and Structures, vol. 14, no. 5, pp. 904-916, 2005.

[11] W. Gao, "Stochastically optimal active control of a smart truss structure under stationary random excitation," Journal of Sound and Vibration, vol. 290, no. 3-5, pp. 1256-1268, 2006.

[12] K. C. Xian, Optimal actuators locations for adaptive structures and structure optimization including discrete variables [Ph.D. thesis], Beihang University, Beijing, China, 2007.

[13] S. Liu and Z. Lin, "Integrated design optimization of voltage channel distribution and control voltages for tracking the dynamic shapes of smart plates," Smart Materials and Structures, vol. 19, no. 12, Article ID 125013, 2010.

[14] S. Honda, I. Kajiwara, and Y. Narita, "Multidisciplinary design optimization for vibration control of smart laminated composite structures," Journal of Intelligent Material Systems and Structures, vol. 22, no. 13, pp. 1419-1430, 2011.

[15] R. Dutta, R. Ganguli, and V. Mani, "Swarm intelligence algorithms for integrated optimization of piezoelectric actuator and sensor placement and feedback gains," Smart Materials and Structures, vol. 20, no. 10, Article ID 105018, 2011.

[16] M. Ahari, Design optimization of an adaptive laminated composite beam with piezoelectric actuators [Dissertation, M.S. thesis], Concordia University, Montreal, Québec, 2005.

[17] W. Y. Wang, Y. J. Wei, C. Wang, and Z. Z. Zou, "Modeling and optimal vibration control of conical shell with piezoelectric actuators," High Technology Letters, vol. 14, no. 4, pp. 418-422, 2008.

[18] W. Gawronski and K. B. Lim, "Balanced actuator and sensor placement for flexible structures," International Journal of Control, vol. 65, no. 1, pp. 131-145, 1996.

[19] Q. Huang, J. Luo, H. Li, and X. Wang, "Analysis and implementation of a structural vibration control algorithm based on an IIR adaptive filter," Smart Materials and Structures, vol. 22, no. 8, Article ID 085008, 2013.

[20] Q. Z. Huang, X. J. Zhu, Z. Y. Gao, S. W. Gao, and E. Y. Jiang, "Analysis and implementation of improved multi-input multioutput filtered-X least mean square algorithm for active structural vibration control," Structural Control and Health Monitoring, vol. 20, no. 11, pp. 1351-1365, 2013. 


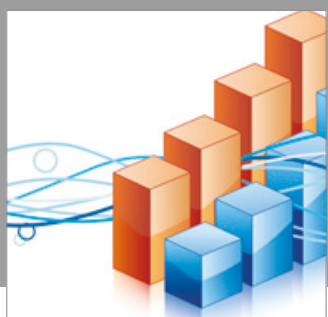

Advances in

Operations Research

vatersals

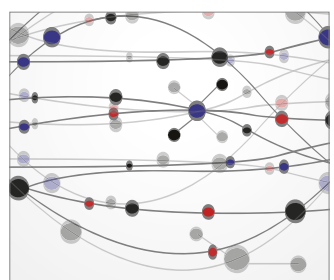

\section{The Scientific} World Journal
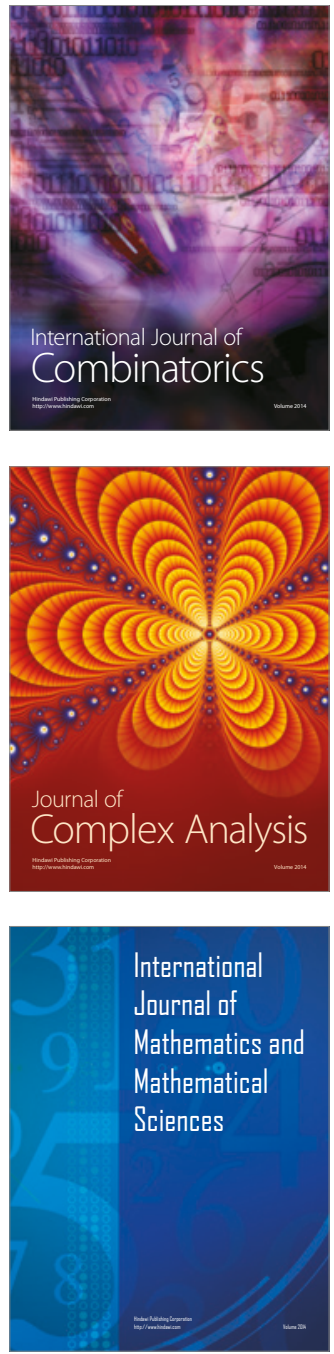
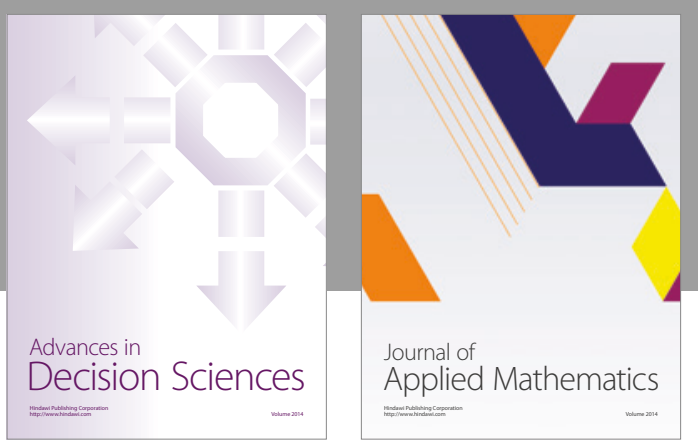

Algebra

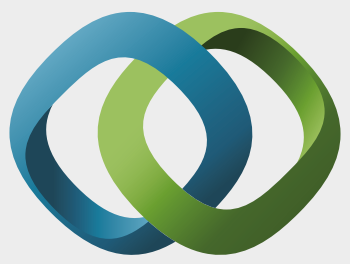

\section{Hindawi}

Submit your manuscripts at

https://www.hindawi.com
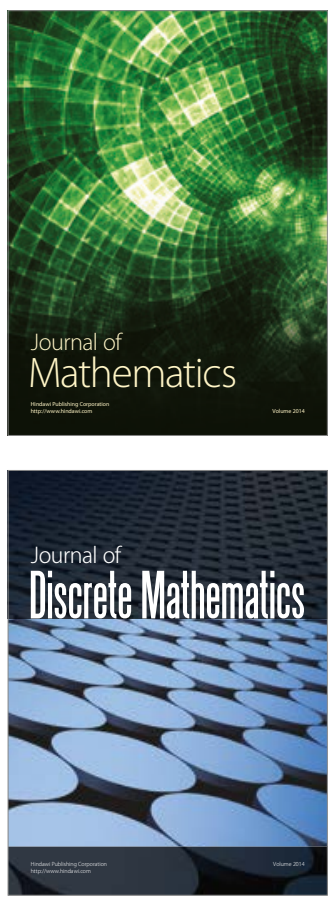

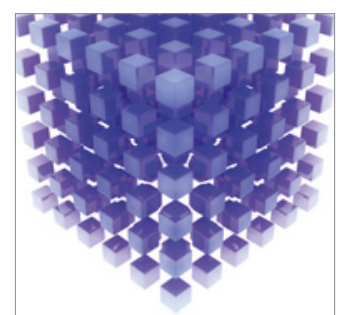

Mathematical Problems in Engineering
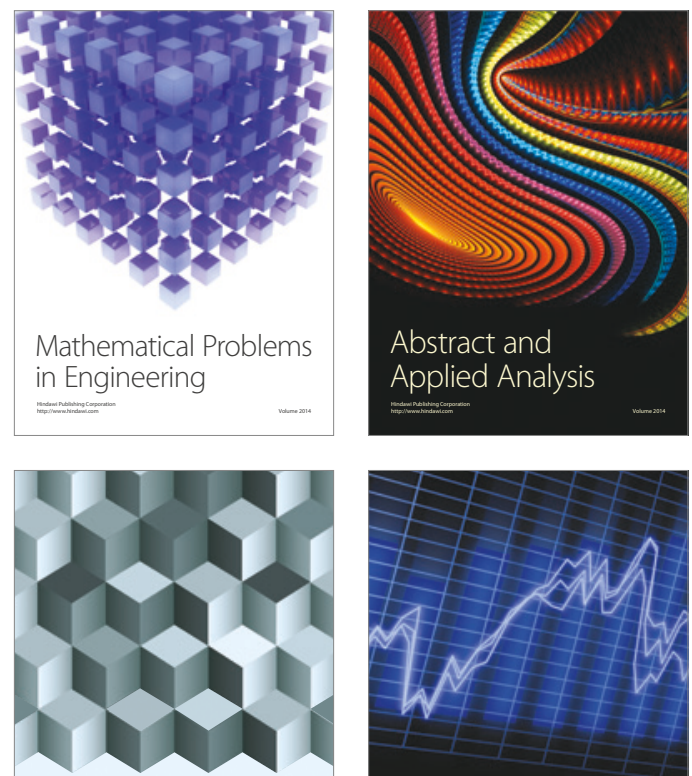

Journal of

Function Spaces

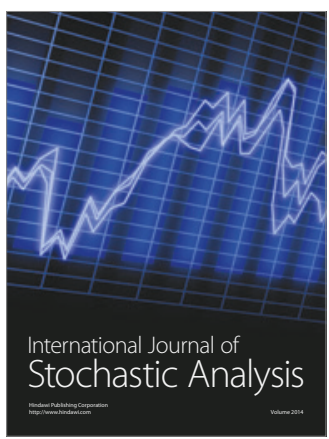

Probability and Statistics
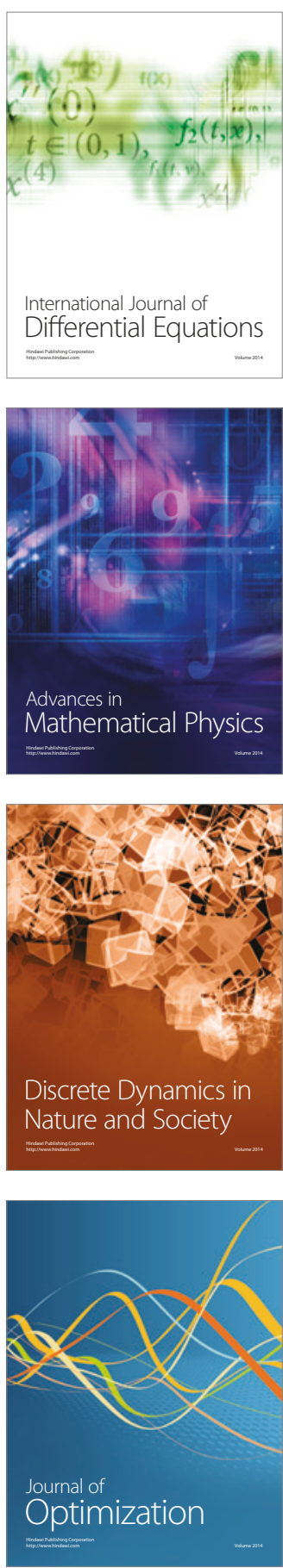